DOI: 10.1002/adma.201405783

Submitted to

Article type: Communication

\title{
Title: Chlorine Resistant Glutaraldehyde Crosslinked Polyelectrolyte Multilayer Membranes for Desalination
}

Kwun Lun Cho, Anita J. Hill, Frank Caruso*, and Sandra E. Kentish*

Dr. Kwun Lun Cho, Prof. Frank Caruso, Prof. Sandra E. Kentish

ARC Centre of Excellence in Convergent Bio-Nano Science and Technology, and the Department of Chemical and Biomolecular Engineering, The University of Melbourne, VIC 3010, Australia

E-mail: fcaruso@unimelb.edu.au, sandraek@unimelb.edu.au

Dr. Anita J. Hill

CSIRO, Materials Science and Engineering, Clayton, VIC 3169, Australia

Keywords: polyelectrolyte multilayer, sulfonated polysulfone, crosslinking, reverse osmosis, desalination

The availability of fresh water for drinking, irrigation and industrial applications is one of the most critical issues today. The production of clean water using membrane technology has been demonstrated as an economical and an energy efficient alternative to current technologies such as thermal distillation. ${ }^{[1]}$ The most commonly used membranes for desalination are thin film composite membranes (TFC) consisting of an active polyamide layer, which provides ion selectivity, deposited onto a porous polysulfone support. ${ }^{[2]}$

The major drawback of the polyamide system is that the membranes are not tolerant to oxidizing agents such as chlorine. Water treatment systems are susceptible to fouling by biological species and the current method of inhibiting such fouling involves the chlorination of the feed water supply. However, the exposure of polyamide membranes to aqueous chlorine has been shown to cause degradation of the aromatic amide bonds, rapidly compromising membrane integrity. ${ }^{[3]}$ Most commercial membrane manufacturers recommend a near-zero exposure of the polyamide membranes to chlorinating agents to prolong membrane lifespan. Current solutions to this issue involve the chlorination of feed water to 


\section{Submitted to

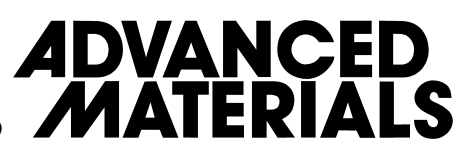

eliminate fouling species followed by de-chlorination upstream of the membrane system. Purified water is then re-chlorinated downstream for distribution. The development of desalination membranes that are chlorine resistant would eliminate these additional processing steps, dramatically reducing operating costs. In the literature, alternative membrane materials (e.g., sulfonated polysulfone) have been shown to exhibit improved chlorine resistance, ${ }^{[4]}$ but none have been commercialized, probably due to the inability for these to be formed into the thin film composite structures that are necessary to provide adequate water permeation.

The layer-by-layer (LbL) assembly of polyelectrolytes allows for the fabrication of separation membranes whereby a wide range of polymers can be readily deposited in aqueous media with a high level of control of properties such as porosity, thickness and composition. ${ }^{[5]}$ These polyelectrolyte multilayer (PEM) membranes have been studied extensively for nanofiltration, forward osmosis and ion selection. ${ }^{[6]}$ However, the low rejection of monovalent ions has been a challenge for the use of PEM membranes in reverse osmosis (RO) applications. To facilitate a reduction in membrane pore size and swelling for greater rejection of monovalent salts, various crosslinking systems have been explored, resulting in reduced membrane swelling and increased ion rejection. ${ }^{[7]}$ Of particular interest, is recent work on the crosslinking of PEM membranes via imine bond formation from the reaction between amines and glutaraldehyde (GA), resulting in highly crosslinked membranes with a high divalent rejection in forward osmosis. ${ }^{[8]}$ However, this study did not quantify rejection of monovalent salts, a crucial property for desalination applications. Furthermore, PEM complexes of polystyrene sulfonate (PSS) and poly(allylamine) hydrochloride (PAH) have been demonstrated in the literature to exhibit resistance to chlorine oxidation, ${ }^{[9]}$ but this property has not been investigated in PEM membranes. 


\section{Submitted to

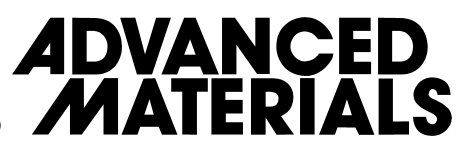

Alternatively, sulfonated polysulfone (sPSf) materials have attracted much interest because of their charged properties and proven chemical resistance. ${ }^{[10]}$ Early reports on the synthesis of sPSf relied on post-polymerization sulfonation methods which produced varied and low levels of sulfonation. However, Wang et al. recently reported the synthesis of sPSf via the polymerization of sulfonated monomers, providing a greater control over the sulfonation level. ${ }^{[11]}$ Desalination membranes fabricated from such polymers have shown remarkable chlorine resistance and good desalination performance. ${ }^{[12]}$ However the hydrophobic nature of the polyethersulfone backbone limits the deposition of the sulfonated polymer to inorganic solvents such as dimethylsulfoxide or dimethylacetamide, making it unsuitable for the polysulfone polymer membrane supports that are currently used.

Herein, we report the assembly of dense polyelectrolyte multilayer membranes, crosslinked via imine bond formation, and their performance in the separation of monovalent $\mathrm{NaCl}$ ions at brackish water concentrations (2000 ppm). The LbL assembly of the widely used polyelectrolytes, PSS and PAH, facilitated the rapid formation of a selective membrane layer with precise control over the thickness and the composition of the membrane. Interlayer crosslinking of $\mathrm{PAH}$ was induced via immersion in GA solution, facilitating imine bond formation. The interlayer crosslinking created a tighter membrane pore size and reduced the membrane swelling. As a result, 10 deposition cycles of PSS/PAH was adequate to form a selective membrane layer with $\mathrm{NaCl}$ rejection of more than $95 \%$. Since only the polycationic layer participates in the crosslinking reaction, the PSS polyanion could then be substituted with another anionic polymer. To this end, a highly sulfonated polysulfone was synthesized and, for the first time, deposited from an aqueous solvent to create a sPSf/PAH PEM assembly. The resultant membrane exhibited similar $\mathrm{NaCl}$ rejection to PSS/PAH membranes, but importantly, showed much greater resistance to chlorine exposure. These results suggest 


\section{Submitted to

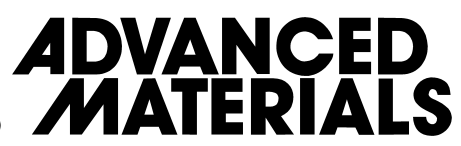

that PEM membranes have outstanding potential for reverse osmosis applications where chlorine resistance is desired.

The formation of the PEMs proceeded with the deposition of a polyethyleneimine (PEI) buffer layer onto a polysulfone support as an adhesion promoter. Subsequently, a layer of PSS was coated onto the surface followed by a layer of PAH, constituting one polyelectrolyte bilayer, as shown in Scheme 1. Up to 10 bilayers were coated onto the supports to ensure uniform coverage of the surface. In between the deposition of each polyelectrolyte layer, the surface was rinsed with Milli-Q water to remove any surface contamination and excess polyelectrolyte, ensuring the formation of a smoother film. The formation of sPSf/PAH multilayer films followed the same procedure. To facilitate film crosslinking, the coated polysulfone supports were immersed in an aqueous GA solution of various concentrations $(0.025 \mathrm{M}$ and $2.5 \mathrm{M})$ for up to $2 \mathrm{~h}$. As depicted in Scheme 1 , the reaction between the amine moieties of $\mathrm{PAH}$ and aldehyde resulted in the formation of imine $(-\mathrm{C}=\mathrm{N}-)$ bonds between and within the PAH layers. The conversion of the primary amine (pKa 9) to imine (pKa 4) during the crosslinking also resulted in an increase in the negative charge of the membrane at neutral $\mathrm{pH}$, as shown in the streaming zeta-potential measurements across the membrane surface (Figure 1). Chemical analysis via ATR-FTIR confirms that imine bond formation occurred within both PSS/PAH and sPSf/PAH membranes (see Supporting Information, Figures S1 and S2). Interestingly, FTIR analysis also highlights the presence of amine and aldehyde signals after the crosslinking reaction, suggesting that the crosslinking of amine moieties was incomplete. This may be attributed to structural densification of the top layers due to the crosslinking reaction, inhibiting diffusion of glutaraldehyde deeper into the membrane. Nevertheless, a significant reduction in the free volume within the membrane film was observed after crosslinking, as demonstrated by the drop in membrane flux shown in Figure 2. Scanning electron microscopy images of the 


\section{Submitted to \\ ADVANCED}

membranes (see Supporting Information, Figure S3) confirm that the bilayers do not penetrate significantly into the porous polysulfone support.

The performance of both the crosslinked and uncrosslinked membranes in separating $\mathrm{NaCl}$ and $\mathrm{MgCl}_{2}$ were evaluated using a dead-end filtration apparatus as a function of membrane bilayer number. As shown in Figure 2a and 2c, a steady increase in the rejection of both monovalent $\mathrm{NaCl}$ and divalent $\mathrm{MgCl}_{2}$ was observed with an increase in the bilayer number. The low rejection and high flux observed on membranes consisting of two bilayers or less is attributed to defects within the films. Subsequently, a steady increase in rejection as a function of bilayer number was observed. A slower rate of increase in both rejection and flux after 6 bilayers could be attributed to the reduced diffusion of glutaraldehyde into the increasingly dense film. A similar trend was observed for sPSf/PAH films. However, a larger variation in ion rejection was observed for sPSf/PAH membranes compared to PSS/PAH membranes. This is attributed to the greater molecular weight distribution in the sPSf oligomer in comparison with PSS, which likely results in variations in pore size distribution and film defects. Despite a significant drop in membrane flux, up to $97 \%$ rejection of monovalent $\mathrm{Na}^{+}$ions was observed.

In both the uncrosslinked and crosslinked PSS/PAH films, the rejection of the divalent $\mathrm{MgCl}_{2}$ was observed to be higher than that of the monovalent ions. For the positively charged, uncrosslinked membrane, this is consistent with ion rejection via the Donnan exclusion mechanism. ${ }^{[13]}$ For the negatively charged crosslinked membranes, the higher rejection of divalent cations over monovalent cations indicates that steric effects are dominant, consistent with the rejection mechanisms proposed for densely crosslinked polyamide membranes. ${ }^{[14]}$ A similar trend was observed for both crosslinked and uncrosslinked sPSf/PAH films indicating, as expected, the same crosslinking mechanism as PSS/PAH. The difference in salt rejection between membranes crosslinked at low and high 


\section{Submitted to

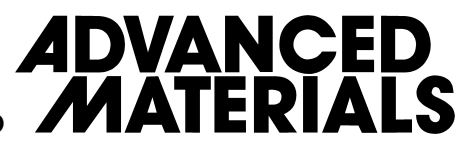

glutaraldehyde concentrations is small, suggesting that crosslinking is efficient at the membrane surface. The minor differences in rejection are attributed to the increasing diffusion of glutaraldehyde into the membrane bulk at higher concentrations. Overall, a higher salt rejection and lower flux was observed for sPSf/PAH films compared to PSS/PAH films, which is attributed to the denser packing and increased hydrophobicity of sPSf compared to PSS.

Membrane flux was also evaluated in a cross-flow filtration setup (Figure 3a) to simulate industrial operation conditions. The fluxes of the fabricated PEM membranes were lower than the commercial TFC membranes, with values of 8 and $6 \mathrm{Lm}^{2} \mathrm{hr}^{-1}$ for PSS/PAH and sPSf/PAH membranes formed with 10 bilayers. However, a direct comparison would be misleading given the level of optimization on the commercial membranes to enhance their performance. More importantly, the present system demonstrates the ability of glutaraldehyde crosslinked films in creating membranes exhibiting high monovalent ion rejection $(90 \%$ and 97\% for PSS/PAH and sPSf/PAH membranes in $2000 \mathrm{ppm}$ of $\mathrm{NaCl}$ at $2400 \mathrm{kPa}$ ). As only the polycation layer contributes to the crosslinking reaction, the system is also significantly more versatile in allowing potentially any anionic polymer (e.g., any sulfonated polymer) to be incorporated into the film without compromising crosslinking density. Alongside the implementation of various optimization techniques reported in the literature, such as the incorporation of polyelectrolyte blends and zeolites, significant improvements in water flux can be foreseen. ${ }^{[15]}$

Both the choice of sPSf as the polyanion and imine bond formation as the crosslinking mechanism indicate increased chlorine resistance. Although imine bonds are readily hydrolyzed at acidic $\mathrm{pH}$ levels, they are resistant to oxidation at neutral and alkaline $\mathrm{pH}$ levels. ${ }^{[16]}$ To evaluate their chemical stability in the presence of chlorine, the membranes were immersed in a solution of sodium hypochlorite at elevated concentrations (3500 ppm) at 


\section{Submitted to

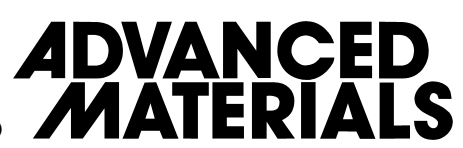

pH 8 to accelerate the effect of chlorine degradation on the membranes. The rejection of $\mathrm{NaCl}$ thereafter was evaluated as a function of chlorine immersion time (ppm-hr) and compared to a commercial polyamide TFC membrane (Dow Filmtec SW30HR). As shown in Figure 3b, the commercial polyamide membranes exhibited a significant reduction in ion rejection after $5 \mathrm{~h}$ of exposure to chlorine. Such observations are consistent with literature values on chlorine degradation of aromatic polyamide RO membranes. The degradation mechanism in aromatic polyamide membrane proceeds via the N-chlorination of the aromatic amine, which ultimately results in polymer chain scission. ${ }^{[17]}$ On the stability of PSS/PAH complexes in aqueous chlorine, previous reports have suggested a combination of reduced electrostatic interaction from the conversion of amine to imine bonds and polymer scission of PSS resulting in leaching of PSS into solution. ${ }^{[18]}$ Chemical analysis (see Supporting Information, Figures S1 and S2) of the PSS/PAH membranes indicates that some degradation of imine bond is observed upon chlorine exposure. However, in the case of sPSf/PAH membranes, no degradation of the imine bonding was observed. As shown in Figure 3b, both PSS/PAH and sPSf/PAH PEM membranes exhibited marked improvements in chlorine resistance compared to the commercial membrane.

In summary, crosslinked polyelectrolyte membranes with high monovalent salt rejection (up to 97\%) have been fabricated. Deposition via spray or dip coating potentially allows for rapid fabrication of new membranes, which can be readily tailored to allow for a wide range of structures for enhanced permeability and selectivity. Synthesis of a highly sulfonated polysulfone allowed for membrane fabrication without the need for organic solvents unsuitable for many commercial membrane supports. Glutaraldehyde crosslinking of the polycationic layers via imine bond formation resulted in a densely packed, negatively charged membrane and, in addition, allowed for the substitution of the polyanion (typically 


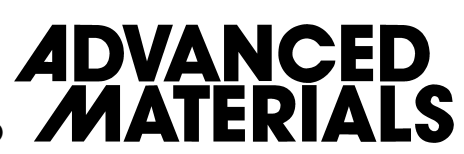

PSS) with sPSf, resulting in increased chlorine resistance with negligible effect on salt rejection.

The resultant membranes exhibited much greater resistance to extended chlorine exposure than commercial aromatic polyamide membranes. GA crosslinked PEM membranes are a promising versatile platform, with readily changeable chemistries, for the fabrication and modification of a filtration membrane ranging from nanofiltration to reverse osmosis membranes. Further optimization with new polyelectrolytes and other additives could offer significantly increased flux, high salt rejection, and further increases in both chemical and biofouling resistance. $^{[19]}$

\section{Experimental Section}

Materials: Bis(4-chlorophenyl) sulfone, bisphenol A, sodium chloride ( $\mathrm{NaCl})$, potassium carbonate $\left(\mathrm{K}_{2} \mathrm{CO}_{3}\right)$, poly(sodium-4-styrene sulfonate) (PSS, $M_{\mathrm{n}} \sim 70$ 000), poly(allylamine hydrochloride) (PAH, $M_{\mathrm{n}} \sim 70$ 000), 30\% fuming sulfuric acid, N-methyl-2pyrolidionone (NMP), N,N-dimethylacetamide (DMA) and toluene were purchased from Sigma-Aldrich and used as received unless specified.

Polysulfone Synthesis: Sulfonated dichlorodiphenyl sulfone (SDCDPS) and sulfonated polysulfone was synthesized in a procedure reported in the literature. ${ }^{[20]}$

Membrane Fabrication: Polyelectrolyte multilayer membranes were assembled on polysulfone substrates (MWCO $\sim 92.5 \mathrm{kDa}, \mathrm{A} 1$, GE Water) by spray-assisted assembly. ${ }^{[21]}$ In a typical process, a $10 \mathrm{ml}$ aliquot of PEI aqueous solution $(1 \mathrm{mg} / \mathrm{ml})$ was sprayed onto the polysulfone membrane surface to act as a primer layer. Subsequently, alternating layers of polyelectrolyte were deposited by spraying $10 \mathrm{ml}$ aliquots of PSS followed by PAH aqueous solutions (1 mg/ml). After each deposition step, a 5 min interval was allowed for polyelectrolyte adsorption, followed by rinsing of excess polyelectrolyte by spraying of the 
membrane surface with water. The spray time for each deposition and/or rinse step was less than 5 s. Crosslinking of the polyelectrolyte multilayer membranes was performed by immersing the membranes into an aqueous solution of glutaraldehyde of various concentrations $(0.025-2.5 \mathrm{M})$ for $2 \mathrm{~h}$.

Membrane Characterization: Attenuated total reflection Fourier transform infrared (ATRFTIR) spectra were collected using a Varian 7000 FT-IR spectrometer (Varian, USA). Each spectrum represents an average of 64 measurements obtained at a wavenumber region of 900 - $4000 \mathrm{~cm}^{-1}$ and resolution of $4 \mathrm{~cm}^{-1}$. Scanning electron microscopy (SEM, Philips XL30, operated at $2 \mathrm{kV}$ ) was used to examine membrane morphology. All samples were goldsputtered before measurements. Membrane surface charge was measured using an AntonPaar Electrokinetic Analyzer (Anton-Paar $\mathrm{GmbH}$, Austria) in a $1 \mathrm{mM} \mathrm{NaCl}$ solution at $\mathrm{pH}$ values ranging from 6 to 9 as adjusted with $\mathrm{NaOH}$. Each data point represents an average of 4 measurements. The streaming-potential was determined using the Helmholtz-Smoluchowski equation. ${ }^{[22]}$ For solute rejection and flux experiments, membranes were cut into $14.6 \mathrm{~cm}^{2}$ coupons for insertion into a HP4750 dead-end filtration cell (Sterlitech, USA). All membranes were compacted overnight at $\sim 6900 \mathrm{KPa}$ in Milli-Q water prior to testing. Flux and solute rejection experiments were conducted at $2400 \mathrm{KPa}$ at ambient temperature (20$\left.25^{\circ} \mathrm{C}\right)$. Single salt rejection experiments were conducted on aqueous $\mathrm{NaCl}(2000 \mathrm{ppm})$ and $\mathrm{MgCl}_{2}$ (2000 ppm) solutions under constant stirring to prevent concentration polarization. An initial clean water flux test was performed before each salt rejection experiment. The mass of permeate collected over time was recorded using an Ohaus Pioneer balance and the flux was calculated from this mass data.

Crossflow filtration experiments were conducted on a rig consisting of three parallel CF042 cells (Sterlitech, USA) as per previously reported. ${ }^{[23]}$ Water flux and salt filtration experiments were conducted on Milli-Q water and $\mathrm{NaCl}$ solutions (2000 ppm), respectively. 


\section{Submitted to

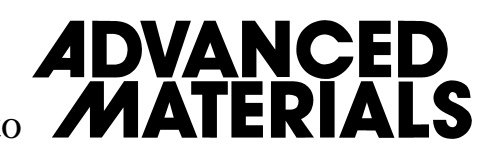

Mass of permeate was collected over a $30 \mathrm{~s}$ time period to calculate flux. Salt rejection was determined from the conductivity of the sample collected. Measurements were repeated 3 times each at 10 min intervals, for three replicate samples and averaged for consistency. Further details of the crossflow rig setup are provided in Supporting Information.

\section{Supporting Information}

Supporting Information is available online from the Wiley Online Library or from the author.

\section{Acknowledgements}

This work was supported by the Australian Research Council Centre of Excellence in Convergent Bio-Nano Science and Technology (Project number CE140100036). The authors acknowledge support from CSIRO under the Science and Industry Endowment Fund. K.L.C. is the recipient of a John Stocker Postdoctoral Fellowship from the Science and Industry Endowment Fund. The authors also thank GE Water for providing the polysulfone membrane substrates used in this work. Dr. Jiwei Cui and Joel Yong are thanked for the SEM imaging.

Received: ((will be filled in by the editorial staff))

Revised: ((will be filled in by the editorial staff)) Published online: ((will be filled in by the editorial staff))

\section{References}

[1] a) M. E. Williams, EET Corporation and Williams Engineering Services Company 2003; b) D. Li, H. Wang, J. Mater. Chem. 2010, 20, 4551; c) L. Malaeb, G. M. Ayoub, Desalination 2011, 267, 1; d) C. Fritzmann, J. Loewenberg, T. Wintgens, T. Melin, Desalination 2007, 216, 1.

[2] a) H. H. Hoehn, ACS Symposium Series (Materials Science of Synthetic Membranes), 1985, 269, 81.; b) J. E. Cadotte, ACS Symposium Series (Materials Science of Synthetic Membranes), 1985, 269, 273.

[3] S. Avlonitis, W. Hanbury, T. Hodgkiess, Desalination 1992, 85, 321.

[4] a) J. Glater, S.-k. Hong, M. Elimelech, Desalination 1994, 95, 325; b) H. B. Park, B. D. Freeman, Z. B. Zhang, M. Sankir, J. E. McGrath, Angew. Chem. 2008, 120, 6108; c) M. Paul, H. B. Park, B. D. Freeman, A. Roy, J. E. McGrath, J. Riffle, Polymer 2008, 49, 2243; d) Y. K. Kim, S. Y. Lee, D. H. Kim, B. S. Lee, S. Y. Nam, J. W. Rhim, Desalination 2010, 250, 865.

[5] a) J. F. Quinn, S. J. Pas, A. Quinn, H. P. Yap, R. Suzuki, F. Tuomisto, B. S. Shekibi, J. I. Mardel, A. J. Hill, F. Caruso, J. Am. Chem. Soc. 2012, 134, 19808; b) R. v. Klitzing, H. Möhwald, Macromolecules 1996, 29, 6901; c) S. T. Dubas, J. B. Schlenoff, Langmuir 2001, 17, 7725; d) R. Steitz, W. Jaeger, R. v. Klitzing, Langmuir 2001, 17, 4471; e) B. Schoeler, G. Kumaraswamy, F. Caruso, Macromolecules 2002, 35, 889; f) A. E. El Haitami, D. Martel, V. Ball, H. C. Nguyen, E. Gonthier, P. Labbé, J.-C. Voegel, P. Schaaf, B. Senger, F. Boulmedais, Langmuir 2009, 25, 2282.

[6] a) S. Qi, C. Q. Qiu, Y. Zhao, C. Y. Tang, J. Membr. Sci. 2012, 405, 20; b) M. L. Bruening, D. M. Sullivan, Chem. Eur. J. 2002, 8, 3832. 


\section{Submitted to

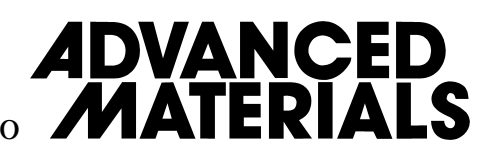

[7] a) J. Park, S. H. Kim, J. Cho, J. Bang, J. Mater. Chem. 2010, 20, 2085; b) C. Qiu, S. Qi, C. Y. Tang, J. Membr. Sci. 2011, 381, 74; c) D. Saeki, M. Imanishi, Y. Ohmukai, T. Maruyama, H. Matsuyama, J. Membr. Sci. 2013, 447, 128.

[8] S. Qi, W. Li, Y. Zhao, N. Ma, J. Wei, T. Wei Chin, C. Y. Tang, J. Membr. Sci. 2012, 423, 536.

[9] a) O. Kreft, R. Georgieva, H. Bäumler, M. Steup, B. Müller-Röber, G. B. Sukhorukov, H. Möhwald, Macromol. Rapid Commun. 2006, 27, 435; b) S. Moya, E. Donath, G. B. Sukhorukov, M. Auch, H. Bäumler, H. Lichtenfeld, H. Möhwald, Macromolecules 2000, 33, 4538; c) W. Tong, C. Gao, H. Möhwald, Chem. Mater. 2005, 17, 4610.

[10] a) A. Noshay, L. Robeson, J. Appl. Polym. Sci. 1976, 20, 1885; b) B. Johnson, I. Yilgör, C. Tran, M. Iqbal, J. Wightman, D. Lloyd, J. McGrath, J. Polym. Sci.: Polym. Chem. Ed. 1984, 22, 721; c) K. Majewska-Nowak, Desalination 1989, 71, 83; d) M. Rucka, G. Pozniak, B. Turkiewicz, W. Trochimczuk, Enzyme Microb. Technol. 1996, 18, 477.

[11] F. Wang, M. Hickner, Q. Ji, W. Harrison, J. Mecham, T. A. Zawodzinski, J. E. McGrath, in Macromol. Symp., Vol. 175, Wiley Online Library 2001, 387.

[12] H. B. Park, B. D. Freeman, Z. B. Zhang, M. Sankir, J. E. McGrath, Angew. Chem. 2008, 120, 6108.

[13] F. G. Donnan, J. Membr. Sci. 1995, 100, 45.

[14] J. Peeters, J. Boom, M. Mulder, H. Strathmann, J. Membr. Sci. 1998, 145, 199.

[15] a) S. G. Kim, D. H. Hyeon, J. H. Chun, B.-H. Chun, S. H. Kim, J. Membr. Sci. 2013, 443; b) L. Sun, R. M. Crooks, J. Am. Chem. Soc. 2000, 122, 12340; c) B. Corry, Energy Environ. Sci. 2011, 4, 751; d) L. Dumée, J. Lee, K. Sears, B. Tardy, M. Duke, S. Gray, J. Membr. Sci. 2012, 427.

[16] R. W. Layer, Chem. Rev. 1963, 63, 489.

[17] a) Y.-N. Kwon, J. O. Leckie, J. Membr. Sci. 2006, 282, 456; b) G.-D. Kang, C.-J. Gao, W.-D. Chen, X.-M. Jie, Y.-M. Cao, Q. Yuan, J. Membr. Sci. 2007, 300, 165.

[18] a) H. C. Beachell, L. H. Smiley, J. Polym. Sci. Part A: Polym. Chem. 1967, 5, 1635; b) S. Moya, L. Dähne, A. Voigt, S. Leporatti, E. Donath, H. Möhwald, Colloids Surf., A 2001, 183, 27.

[19] a) J. Haldar, D. An, L. Alvarez de Cienfuegos, J. Chen, A. M. Klibanov Proc. Natl. Acad. Sci. U.S.A. 2006, 103, 17667. b) J. Frueh, M. Gai, Z. Yang, Q. He, J. Nanosci. Nanotechnol. 2014, 14, 4341. c) D. Park, J. Wang, A. M. Klibanov, Biotechnol. Progress 2006, 22, 584.

[20] W. L. Harrison, F. Wang, J. B. Mecham, V. A. Bhanu, M. Hill, Y. S. Kim, J. E. McGrath, J. Polym. Sci. Part A: Poly. Chem. 2003, 41, 2264.

[21] a) J. B. Schlenoff, S. T. Dubas, T. Farhat, Langmuir 2000, 16, 9968; b) K. L. Cho, H. Lomas, A. J. Hill, F. Caruso, S. E. Kentish, Langmuir 2014, 30, 8784.

[22] E. Menachem, A. E. Childress, Water Treatment Technology Program Report No. 10. Bureau of Reclamation, Denver Office, 1996.

[23] K. Kezia, J. Lee, A. Hill, S. Kentish, J. Membr. Sci. 2013, 445, 160. 


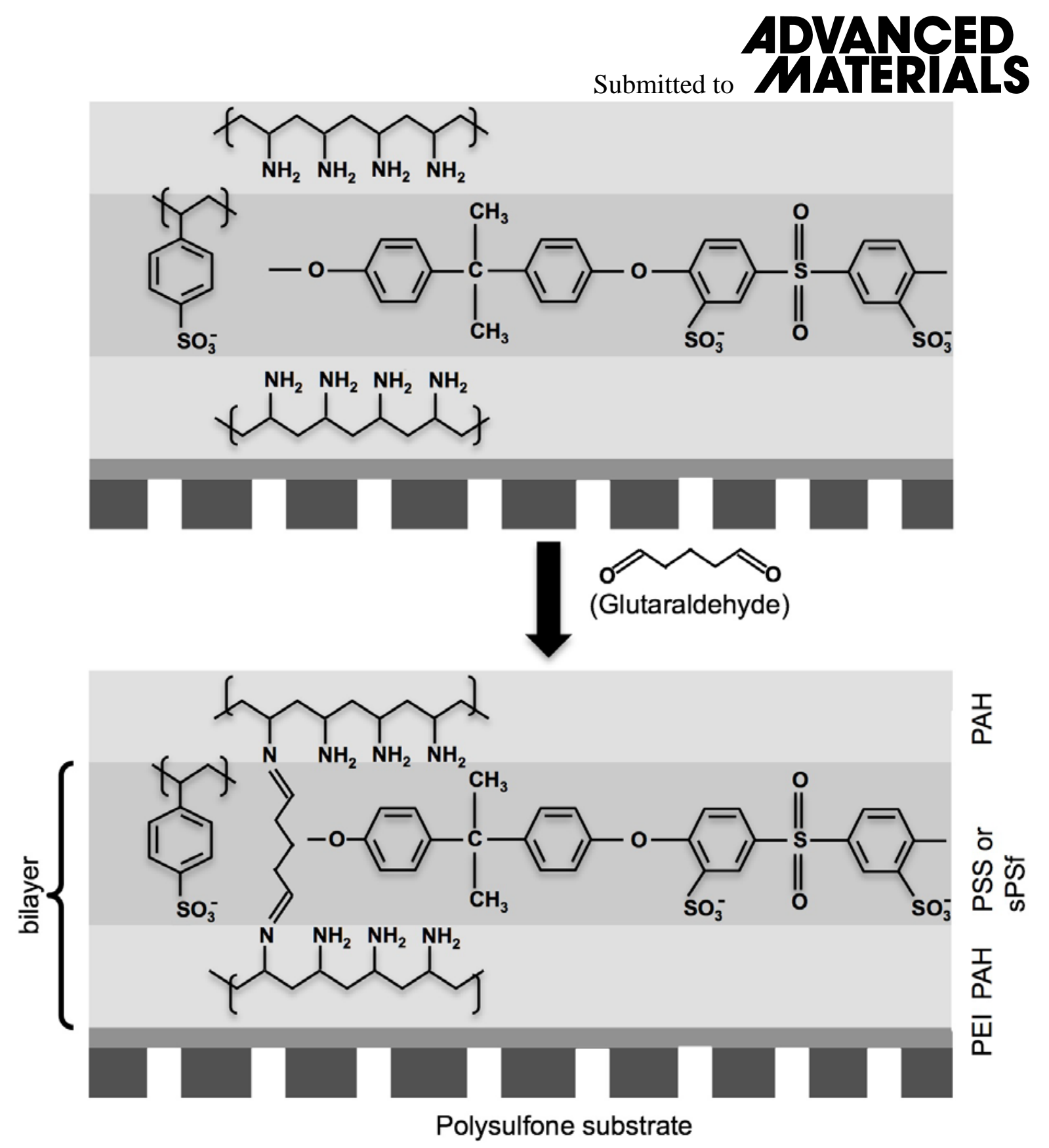

Scheme 1. Schematic illustration of a PEM film, with either PSS or sPSf in the polyanionic layer, before and after glutaraldehyde crosslinking. 


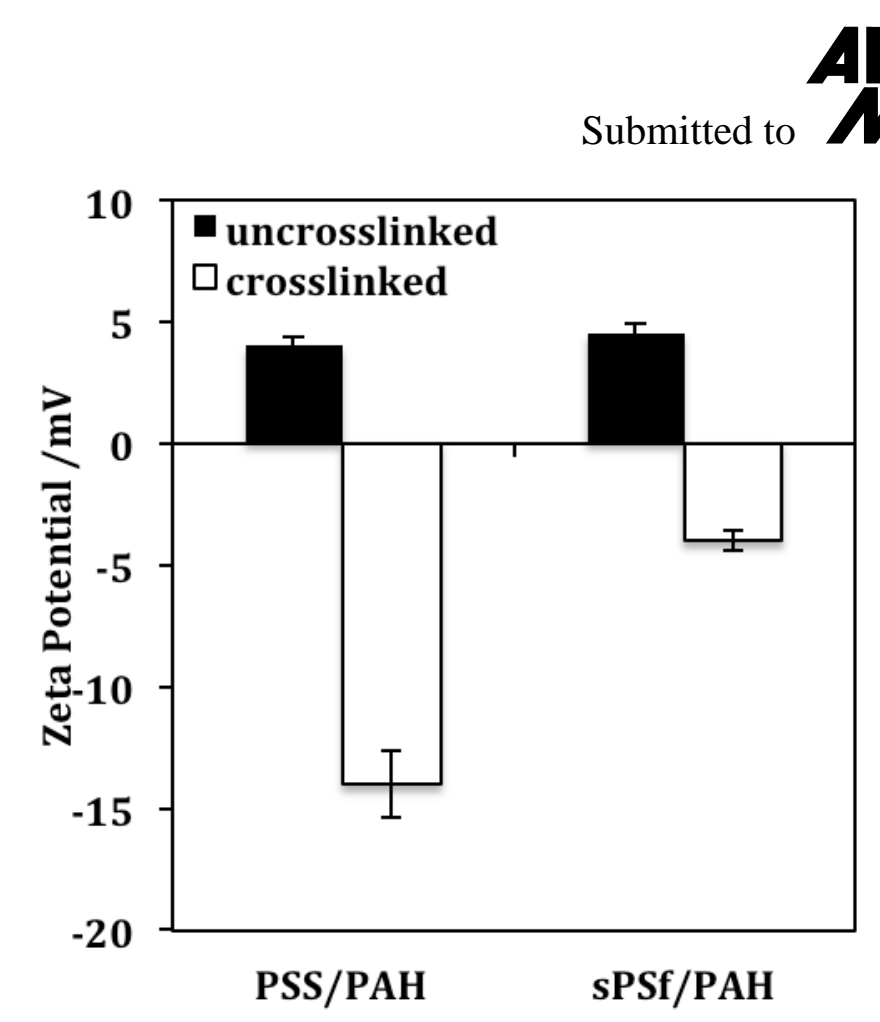

Figure 1. Streaming zeta potential measurement of PSS/PAH and sPSf/PAH membranes at pH 7.5, before and after GA crosslinking. 


\section{Submitted to}
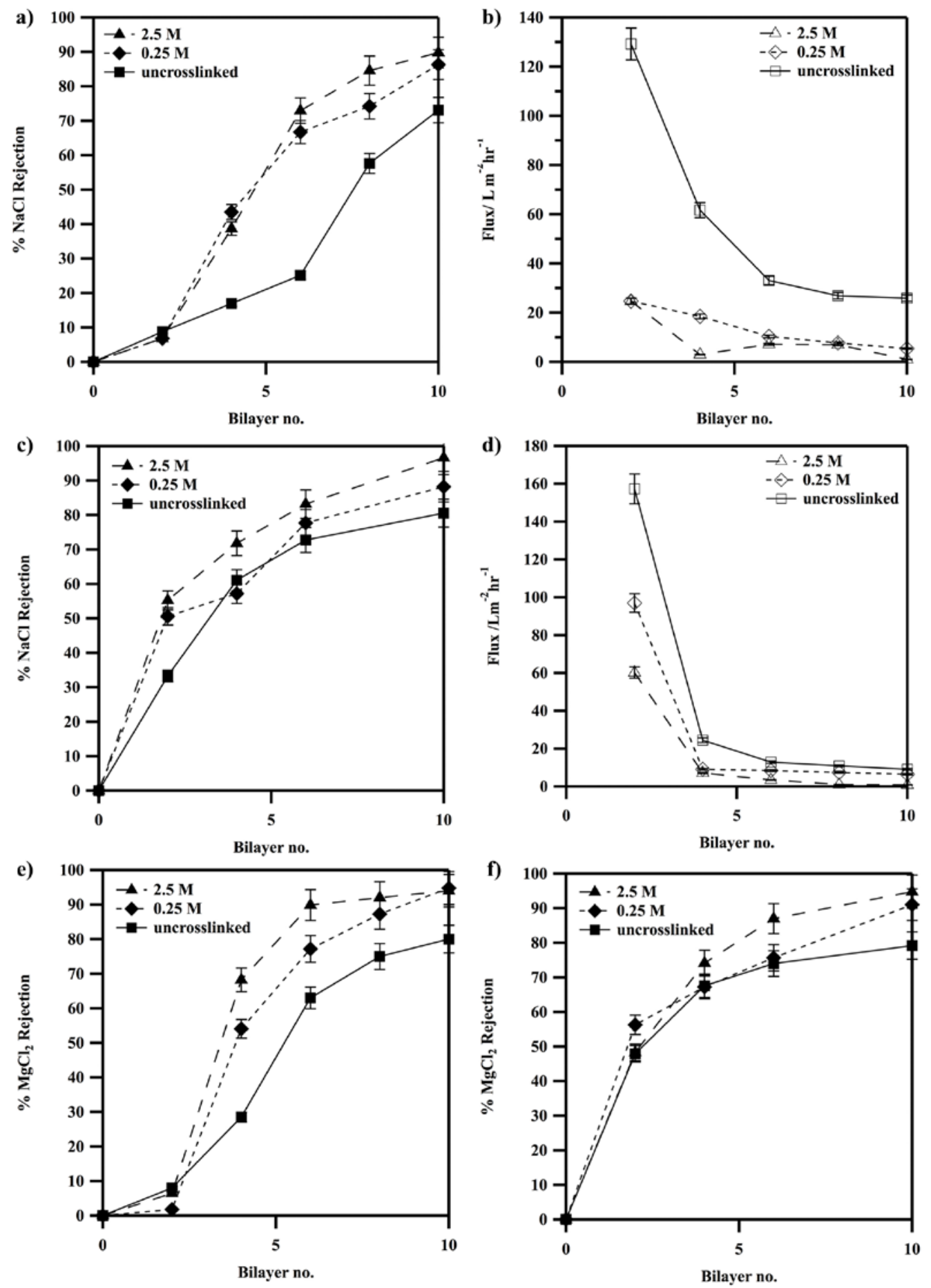

Figure 2. a) $\mathrm{NaCl}$ rejection and b) Flux of PSS/PAH membranes at various crosslinker concentrations as a function of bilayer number. c) $\mathrm{NaCl}$ rejection and d) Flux of sPSf/PAH membranes crosslinker concentrations as a function of bilayer number. $\mathrm{MgCl}_{2}$ rejection of $\left.\mathbf{e}\right)$ PSS/PAH and f) sPSf/PAH membranes at various crosslinker concentrations. Permeation experiments conducted at $2400 \mathrm{KPa}$ transmembrane pressure and $2000 \mathrm{ppm} \mathrm{NaCl}$. 


\section{Submitted to
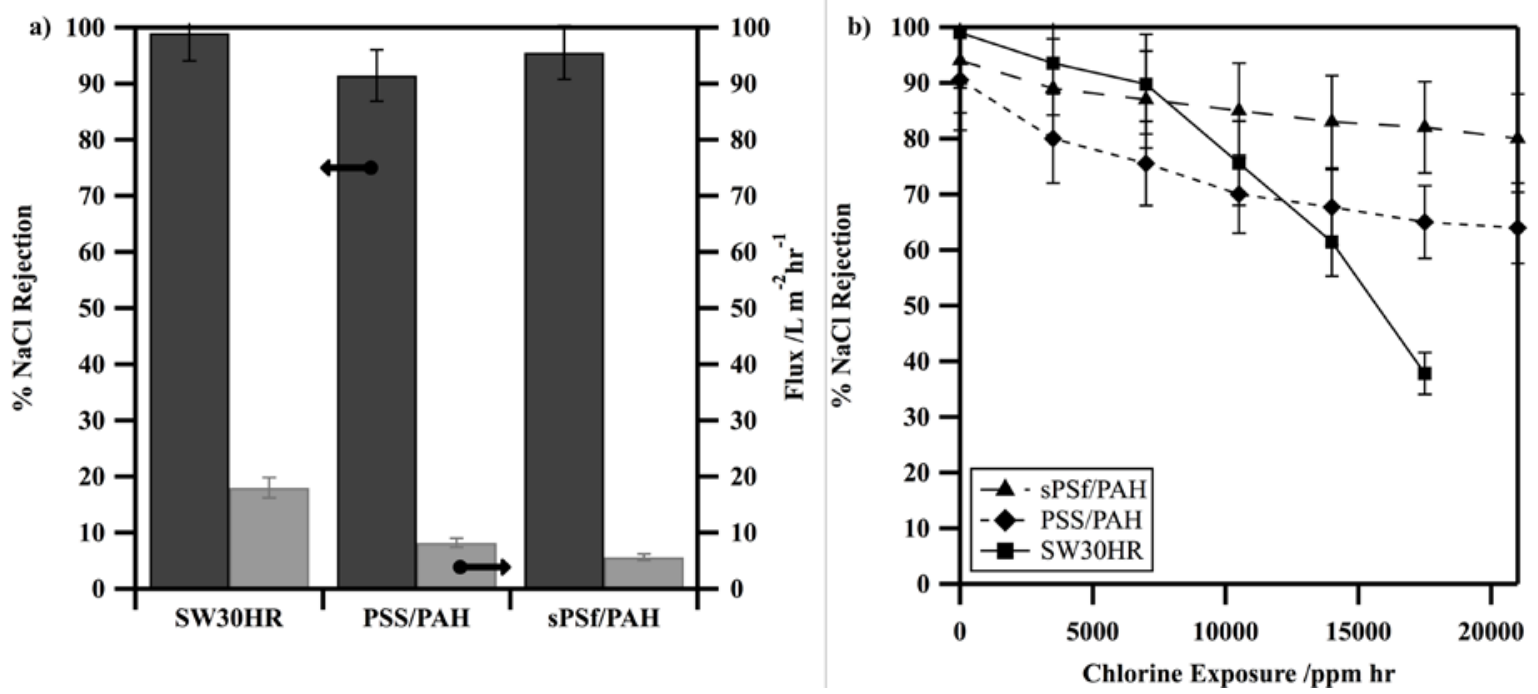

Figure 3. a) $\mathrm{NaCl}$ rejection and flux of crosslinked membranes in cross-flow experiments and b) $\mathrm{NaCl}$ rejection of crosslinked membranes as a function of chlorine exposure time, in comparison to commercial SW30HR membrane. Permeation experiments conducted at 2400 KPa transmembrane pressure and 2000 ppm NaCl. 
Submitted to

\section{ADVANCED}

The table of contents entry should be 50-60 words long (max. 400 characters), and the first phrase should be bold. The entry should be written in the present tense and impersonal style.

Keyword polyelectrolyte multilayer, sulfonated polysulfone, crosslinking, reverse osmosis, desalination

Kwun Lun Cho, Anita J. Hill, Frank Caruso*, and Sandra E. Kentish* ((same order as byline))

Title : Chlorine Resistant Glutaraldehyde Crosslinked Polyelectrolyte Multilayer Membranes for Desalination

Crosslinked polyelectrolyte multilayer membranes are synthesized with salt rejection approaching that of commercial desalination membranes, but with major improvements in chlorine resistance. The membranes are fabricated within aqueous media onto porous commercial substrates. Crosslinking of the polycation layers with glutaraldehyde leads to $\mathrm{NaCl}$ rejections of up to $97 \%$, while the incorporation of a highly sulfonated polysulfone polyanion leads to outstanding chlorine resistance.

ToC figure ((Please choose one size: $55 \mathrm{~mm}$ broad $\times 50 \mathrm{~mm}$ high or $110 \mathrm{~mm}$ broad $\times 20 \mathrm{~mm}$ high. Please do not use any other dimensions))

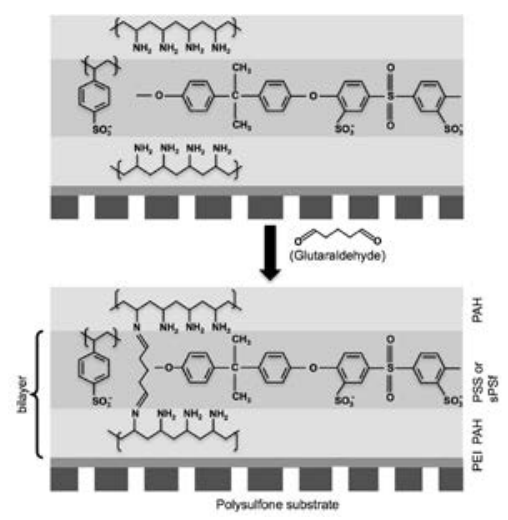

